\title{
CORONARY ARTERY DISEASE
}

\section{Stable CAD does not SIGNIFY ivabradine therapy}

An elevated heart rate is a well-established marker of cardiovascular risk, but treatment with ivabradine to decrease heart rate does not improve outcomes in patients with stable coronary artery disease (CAD). This finding comes from the SIGNIFY trial and was presented at the ESC Congress 2014. Ivabradine is an inhibitor of the $I_{\mathrm{f}}$ or 'pacemaker' current in the sinoatrial node and has been shown to reduce heart rate without affecting blood pressure or left ventricular systolic function.

Investigators recruited 19,102 patients with stable CAD, no clinical heart failure, and a heart rate $\geq 70 \mathrm{bpm}$. Patients were randomly allocated to ivabradine or placebo, in addition to standard background therapy. After 3 months, heart rate was $60.7 \pm 9.0 \mathrm{bpm}$ in the ivabradine-treated group compared with $70.6 \pm 10.1 \mathrm{bpm}$ in the group who received placebo. Despite this reduction in heart rate, no significant difference was observed between ivabradine treatment and placebo in the primary composite end point of death from cardiovascular causes and nonfatal myocardial infarction $(6.8 \%$ versus 6.4\%; HR 1.08, 95\% CI 0.96-1.20, $P=0.20$ ). Moreover, in a subgroup analysis, ivabradine was associated with an increase in the primary end point in patients with activity-limiting angina. Ivabradine was also associated with an increased incidence of bradycardia $(18.0 \%$ vs $2.3 \% ; P<0.001)$.

The investigators conclude that "these results suggest that an elevated heart rate is only a marker of risk-but not a modifiable determinant of outcomes -in patients who have stable CAD without clinical heart failure".

Gregory B. Lim

Original article Fox, K. et al. Ivabradine in stable coronary artery disease without clinical heart failure. N. Engl. J. Med. doi:10.1056/NEJMoa1406430 